\title{
Adrenal incidentalomas: imaging challenges-role of MDCT scan versus MRI in evaluating adrenal incidentalomas
}

\author{
Monica Maher Amin Nawar', Sameh Abdel Aziz Zaky Hanna', Shereen Sadik El-Sawy ${ }^{2}$ and Sally Yehia Shokralla ${ }^{1 *}$ (D)
}

\begin{abstract}
Background: The term adrenal incidentaloma (Al), by definition, is an adrenal mass that is unexpectedly detected through an imaging procedure performed for reasons unrelated to adrenal dysfunction or suspected dysfunction. Despite their frequent appearance, the challenge remains in recognizing and treating the small percentage of $\mathrm{Al}$ that poses a significant risk, either because of their hormonal activity or because of their malignant histology. The aim of this study is to study the role of MRI, specifically chemical shift imaging (CSI), against various MDCT scans (non-enhanced, enhanced, and delayed) in the characterization of incidentally discovered adrenal masses to offer a way for the patients to avoid unnecessary time and money-wasting imaging modalities used to reach a diagnosis of their incidentally discovered adrenal lesions. We examined a total number of 20 patients with total of 22 adrenal lesions. The mean age was $51.1 \pm 15.27$.
\end{abstract}

Results: In our study, we found that among CT parameters, APW and RPW showed the highest sensitivity and specificity for detection of lipid-rich adenomas. CSI has also proven to be the best MR technique. However, there is no statistically significant difference in the diagnostic capability of CSI versus the CT washout technique. Both modalities could be conducted, according to specific patient preferences and/or limitations, with comparable highly accurate outcomes.

Conclusion: This study demonstrates that a similar diagnostic outcome is obtained from contrast-enhanced CT (CECT) and MRI with CSI of adrenal lesions.

Keywords: Adrenal, Adrenal mass, Adrenal adenoma, Incidentalomas, Chemical shift imaging

\section{Background}

Adrenal masses discovered accidentally in the course of imaging for other abdominal conditions are known as adrenal incidentalomas (AIs). Those patients who are having these incidentalomas-by definition-have no history or symptoms of adrenal diseases prior to imaging procedures that lead to their discovery [1]. Adrenal metastasis, which is a common radiological finding in 6$20 \%$ of patients with extra-adrenal malignancy, should not be considered AI [2].

\footnotetext{
* Correspondence: sallykozman@yahoo.com

'Department of Radiodiagnosis, Faculty of Medicine, Cairo University, Cairo, Egypt

Full list of author information is available at the end of the article
}

The prevalence of AI increases with age. It reaches up to $7 \%$ of people above the age of 70 compared to only $0.5 \%$ of people in their $20 \mathrm{~s}$. On the other hand, AIs are present in $4-5 \%$ of abdominal CT examinations [3].

Adrenal incidentalomas comprise a wide range of pathologies but the vast majority of cases are adrenal adenomas. Although in most cases, this is not proved pathologically, yet the clinical course can depict its benign nature. However, still there is a percentage of these AIs which could be either malignant or hormone secreting lesions which are not clinically evident yet. Table 1 demonstrates imaging findings in common adrenal pathologies. Based on that, both the physicians and the radiologists should do every effort to exclude or prove the non-benign nature of these 
Table 1 Imaging findings differentiating common adrenal pathologies in Als

\begin{tabular}{|c|c|c|c|c|}
\hline Finding & Benign adenoma & ACC & Pheochromocytoma & Metastases \\
\hline Size & Mostly less than $4 \mathrm{~cm}$ & Mostly greater than $4 \mathrm{~cm}$ & Variable & Variable \\
\hline Growth rate & $\begin{array}{l}\text { Stable or slow growth }(<0.8 \\
\mathrm{cm} / \text { year })\end{array}$ & $\begin{array}{l}\text { Significant growth (> } 1 \\
\mathrm{~cm} / \text { year) }\end{array}$ & Slow growth & $\begin{array}{l}\text { Significant growth (> } 1 \\
\mathrm{~cm} / \text { year) }\end{array}$ \\
\hline Shape and margins & $\begin{array}{l}\text { Round or oval with well- } \\
\text { defined margins }\end{array}$ & $\begin{array}{l}\text { Irregular shape and } \\
\text { margins. }\end{array}$ & Variable & Variable \\
\hline Composition & Homogenous & $\begin{array}{l}\text { Heterogeneous } \\
\text { (hemorrhage, necrosis) }\end{array}$ & Heterogeneous (necrosis) & $\begin{array}{l}\text { Heterogeneous } \\
\text { (hemorrhage, necrosis) }\end{array}$ \\
\hline $\begin{array}{l}\text { CT unenhanced } \\
\text { attenuation }\end{array}$ & $\begin{array}{l}\leq 10 \mathrm{HU} \text { (or }>10 \mathrm{HU} \text { for lipid- } \\
\text { poor adenomas) }\end{array}$ & $>10 \mathrm{HU}$ & $>10 \mathrm{HU}$ & $>10 \mathrm{HU}$ \\
\hline $\begin{array}{l}\text { CT percent } \\
\text { washout }(P W)\end{array}$ & $\mathrm{APW}>60 \%, \mathrm{RPW}>40 \%$ & APW $<60 \%$, RPW $<40 \%$ & APW $<60 \%$, RPW $<40 \%$ & APW $<60 \%$, RPW $<40 \%$ \\
\hline $\begin{array}{l}\text { MRI-CSI (out-of } \\
\text { phase) }\end{array}$ & $\begin{array}{l}\text { Signal loss (except in lipid-poor } \\
\text { adenomas) }\end{array}$ & $\begin{array}{l}\text { No change in signal } \\
\text { intensity }\end{array}$ & No change in signal intensity & $\begin{array}{l}\text { No change in signal } \\
\text { intensity }\end{array}$ \\
\hline FDG uptake (PET) & $\begin{array}{l}\text { Low (some can have low to } \\
\text { moderate uptake) }\end{array}$ & High & $\begin{array}{l}\text { Low (malignant pheochromocytomas } \\
\text { show high uptake) }\end{array}$ & High \\
\hline
\end{tabular}

ACC adrenocortical carcinoma, HU Hounsfield units, APW absolute PW, RPW relative PW, CSI chemical-shift imaging, FDG fluoro-deoxyglucose [Chatzellis and Kalsas, 2019]

lesions such as adrenocortical carcinoma (ACC) as well as other AI with secretory potentials [2].

The main target in dealing with AI is to prove or exclude the presence of malignancy especially ACC or the presence of secretory potential. This entails careful clinical history and examination, biochemical testing, and detailed radiographic examination.

Careful clinical history and examination might reveal symptoms and signs of adrenal dysfunction in hormone secreting AI, and this needs high index of suspicion among physicians [4].

Currently, CT scan and MRI examination are the imaging modalities of choice with high diagnostic accuracy regarding the depiction of the nature of the lesion and the presence of malignant potential. The size of the lesion is an important determinant of the malignant potential of AI as the ACC is larger than adenomas. The risk of malignancy increases with the size, in one meta-analysis it was found that ACCs represent $2 \%$ of all tumors $<4 \mathrm{~cm}$ and up to $25 \%$ in tumors $>6 \mathrm{~cm}$ [5]. Definitely, the size alone has low specificity in diagnosis of malignant potential. Other criteria, which can suggest the malignant potential, include irregular shape and borders, tumor heterogeneity with central necrosis or hemorrhage and invasion into surrounding structures. On the other hand, the main features of benign adrenal adenomas are their smaller size, regular outline, and slower growth [2].

There are some imaging properties of AIs, depending on the modality used, which can be helpful for differentiation between benign and malignant adrenal lesions are shown in (Table 1).

\section{Methods}

This cross-sectional analytic study was conducted on 20 patients at the Radiology Department in collaboration with the Internal Medicine Department using MDCT and MRI. This was done after approval from the institutional review board and obtaining informed consent from all patients. The mean age of the participants was $51.1 \pm 15.27$ with 2:3 female to male ratio. Twenty patients were included in our study with a total number of 22 lesions.

\section{Inclusion criteria}

Adult patients with accidentally discovered adrenal lesions by a previous imaging modality.

\section{Exclusion criteria}

People performed adrenal surgical procedures, people known to have known endocrine problems, pediatric age group, and pregnant patients are all excluded. Claustrophobic patients were excluded as well.

The following measures have been done:

Informed written consent with detailed explanation of the study and its purpose was obtained from each patient. This is followed by an inquiry of all patients about how they discovered their adrenal lesion(s) and whether they performed subsequent laboratory tests or not.

Unenhanced and if possible, contrast-enhanced CT and MRI protocols (T1-T2-DUAL phase and DWI) for characterizing their adrenal lesions. Six months and/or 12 months follow-up CT and MRI studies were performed by contacting the patients' phones and arranging meeting or through their treating physician. Also, a histopathological confirmation was obtained if available. A combination of pathological results and/or follow-up was considered as a standard of reference. 


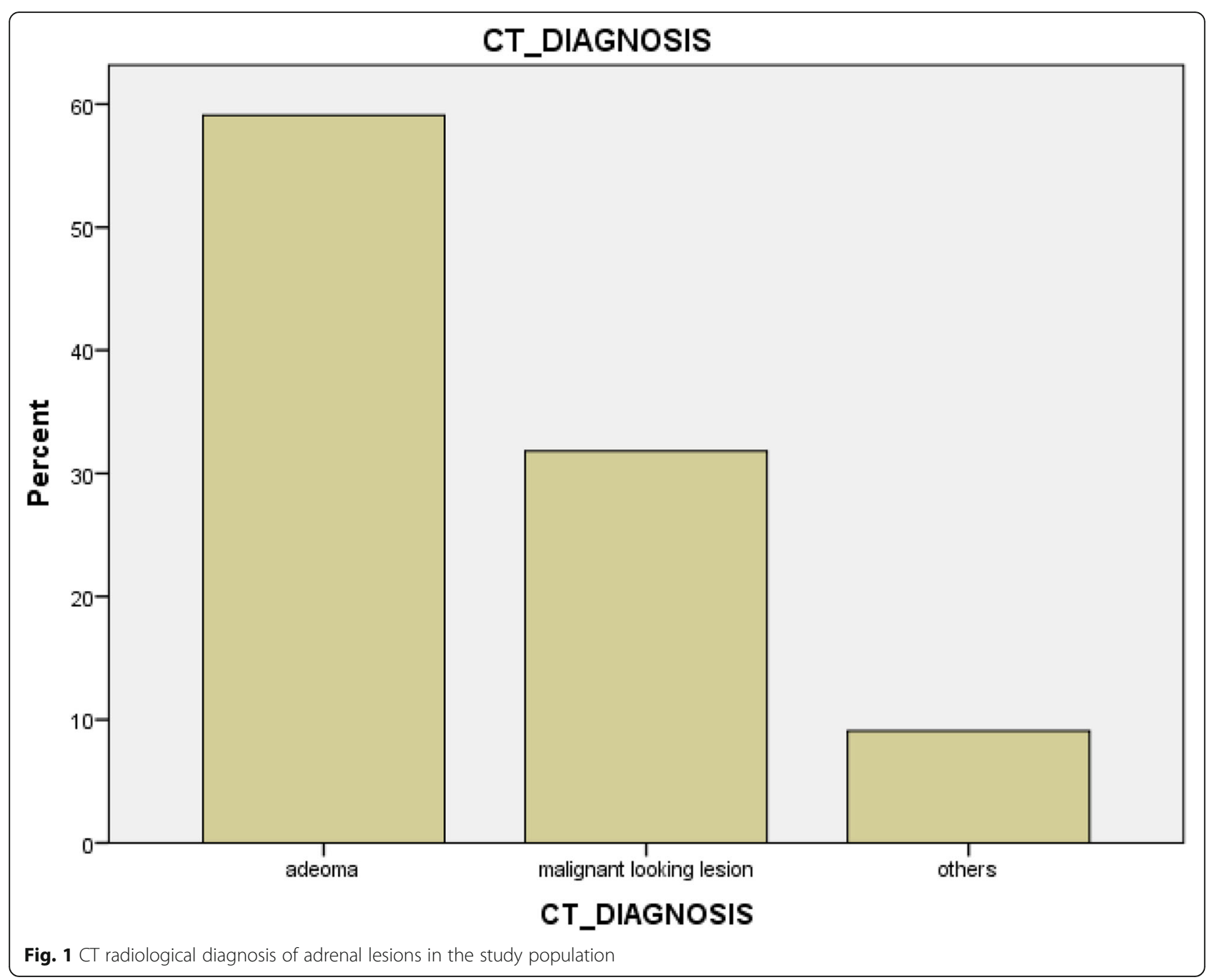

\section{Protocol for MDCT}

MDCT imaging of the abdomen, of 20 patients with 4 of them failed to endure contrast due to raised creatinine level, was performed with a multi-detector row helical CT scanner (GE; Medical systems). Imaging parameters included section collimation of 3-5 mm, high-quality mode, table speed of $7.5 \mathrm{~mm} / \mathrm{s}, 50 \%$ overlap reconstruct. The first set of images (precontrast phase) was taken before intravenous contrast administration. The second set (portal venous phase) was taken $75 \mathrm{~s}$ after intravenous injection of $120 \mathrm{ml}$ of contrast medium. Delayed images,

Table 2 MRI diagnosis of adrenal lesions in study population

\begin{tabular}{lll}
\hline & Frequency & Percent \% \\
\hline Lipid-rich adenoma & 13 & 59.1 \\
Malignant looking lesion & 7 & 31.8 \\
Others & 2 & 9.1 \\
total & 22 & 100 \\
\hline
\end{tabular}

the last set of images, were obtained after $15 \mathrm{~min}$ from the time of injection using $120 \mathrm{KV}$ and $280-380 \mathrm{~mA}$.

\section{Post-processing calculations}

We had three sets of images: pre attenuation (PA), early contrast enhanced, early attenuation (EA), and delayed contrast enhanced, delayed attenuation (DA) images. All these images were recorded. A circular region of interest, ROI was placed in the center of the adrenal mass on the section where it appeared the largest. The ROI covered approximately half to two-thirds of the mass excluding any region of calcification or degeneration, which luckily was not common in our cases. Then, absolute percentage washout (APW) and the relative percentage washout (RPW) were calculated using the following formulae:

$$
\begin{aligned}
& \mathrm{APW}=[(\mathrm{EA}-\mathrm{DA}) /(\mathrm{EA}-\mathrm{PA})] \times 100 \text { and } \\
& \mathrm{RPW}=[(\mathrm{EA}-\mathrm{DA}) / \mathrm{EA}] \times 100[6]
\end{aligned}
$$




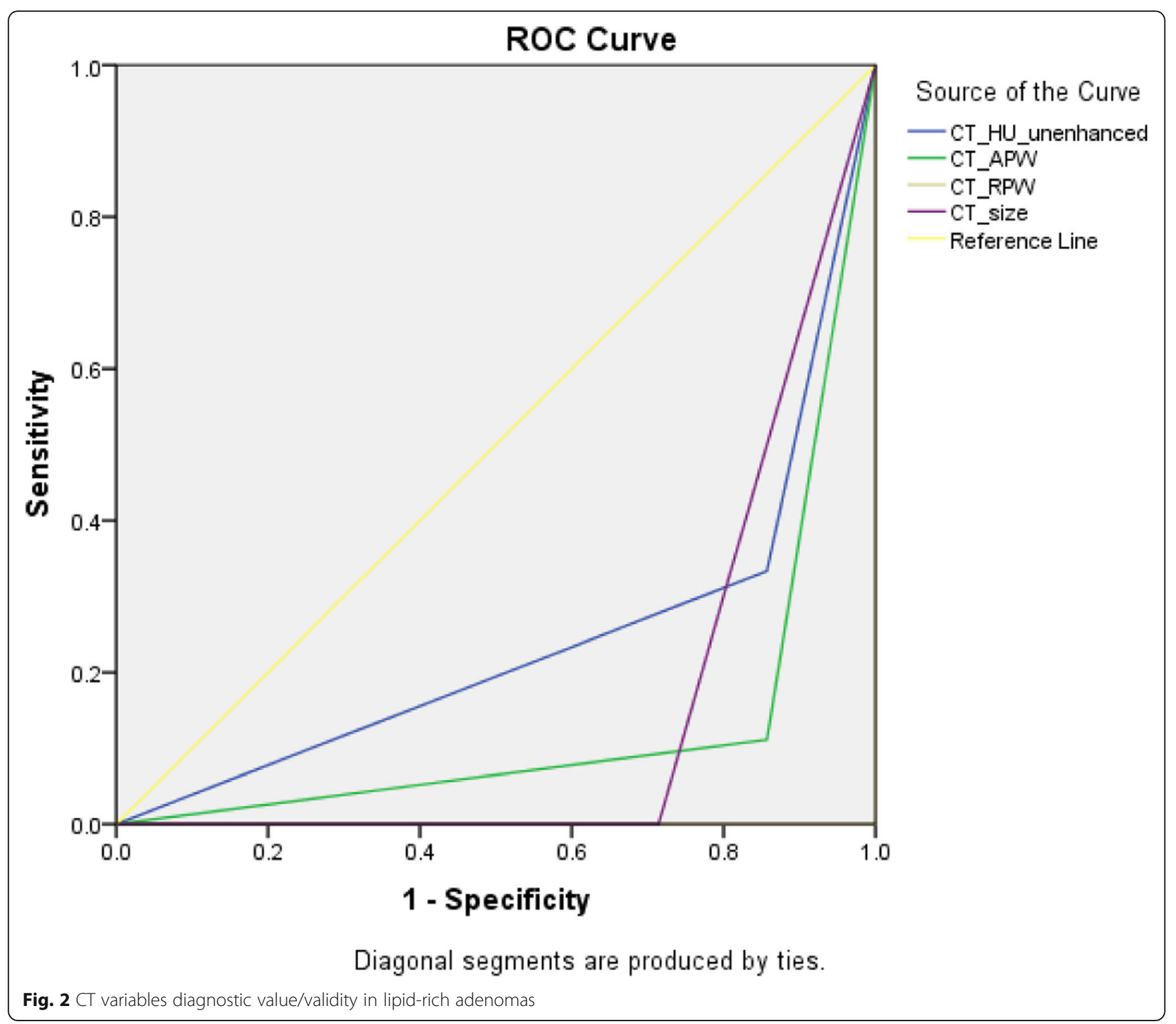

\section{Protocol for MRI}

MR imaging was performed in 20 patients using a $1.5 \mathrm{~T}$ unit (Philips GyroscanIntera) with a phased-array body coil. T1-weighted spin echo axial images were acquired with a TR of $425 \mathrm{~ms}$ and a TE of $15 \mathrm{~ms}$. T2-weighted

Table 3 Area under the curve of CT diagnostic value in benign lesions

\begin{tabular}{lc}
\hline Test result variable(s) & Area \\
\hline CT_HU_unenhanced & .238 \\
CT_APW & .127 \\
CT_RPW & .000 \\
CT_size & .143
\end{tabular}

As seen in Fig. 3, the ROC curve, and Table 4, area under the curve, we conclude that the MRI CSI and T2 signal are best indicators used for MRI detecting intracytoplasmic fat content and confirming high lipid content in lipid-rich adenomas axial and coronal images were acquired with the fast spin echo technique using a TR of $1800 \mathrm{~ms}$ and a TE of $100 \mathrm{~ms}$.

The section thickness was $4 \mathrm{~mm}$ with an intersection gap of $0.4 \mathrm{~mm}$ for both T1- and T2-weighted imaging. The matrix size was $204 \times 256$. Respiratory gating was used for spin echo imaging with flow compensation being used for most of the spin echo images.

\section{Chemical shift imaging (CSI)}

To obtain the chemical shift imaging (CSI), we used a breath-holding T1-weighted gradient recalled echo sequence, a TR of $68-160 \mathrm{~ms}$, a TE of $4.9 \mathrm{~ms}$ for in-phase images, and a TE of $6.3 \mathrm{~ms}$ for opposed-phase images. The flip angle was $30^{\circ}$, the matrix size $128 \times 256$, and the field of view varied from 320 to $375 \mathrm{~cm}$. 


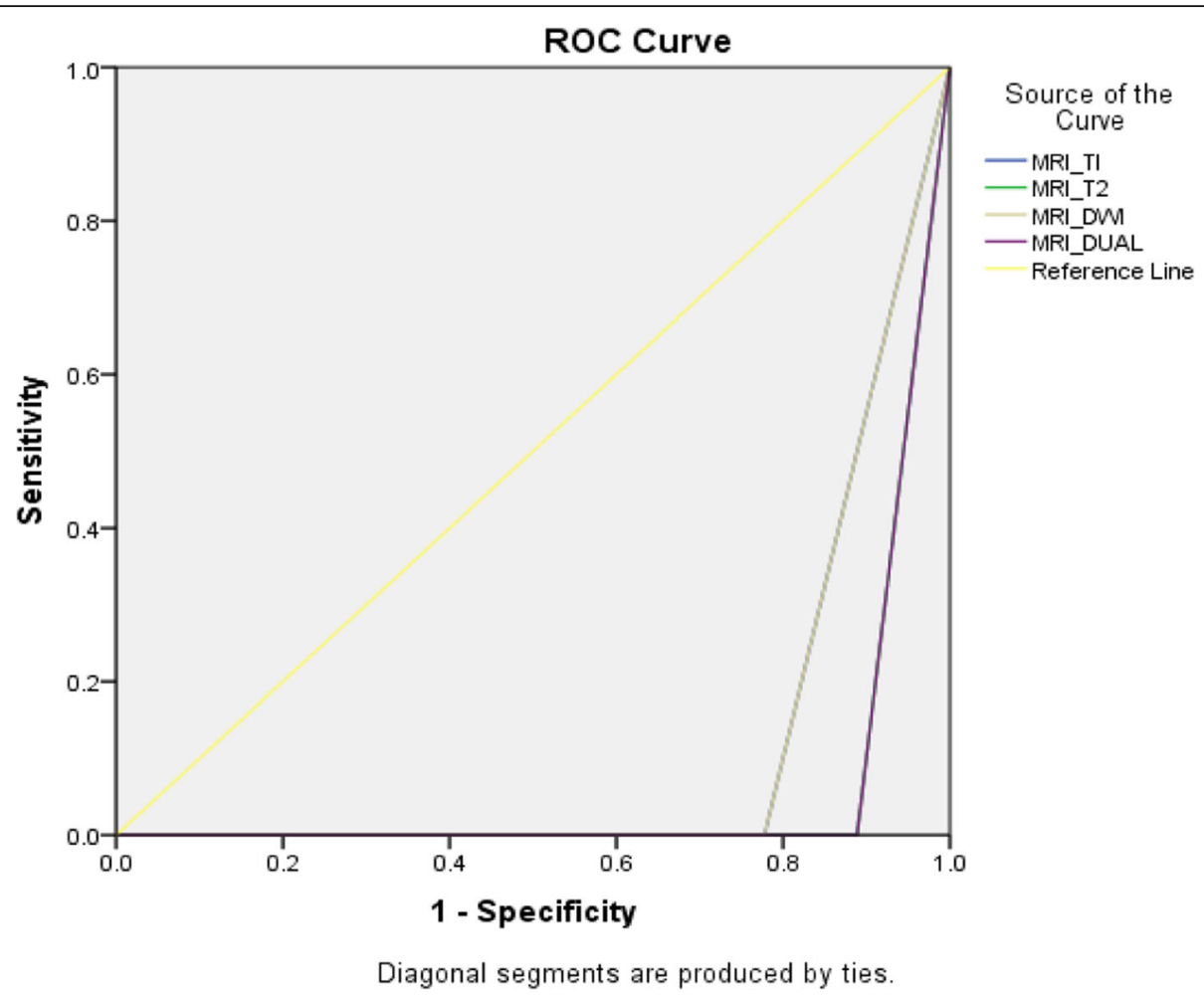

Fig. 3 MRI sequences diagnostic value/validity in lipid-rich benign lesion

The obtained chemical shift images were qualitatively assessed to find out if there was significant drop of signal in the lesion on the opposed-phase images compared to the in-phase images or not [7].

\section{Statistical data analysis}

Data analysis packages are SPSS version 21 and Medcalc. Qualitative data was presented by number and percentage, while quantitative data was presented by mean, standard deviation.

Statistical tests were done for qualitative data: chisquare test with Fisher's exact. Level of significance was considered if $p$ equal to or below 0.05 .

ROC curve was used to detect predictive value of MRI chemical shift and MDCT in adrenal incidentalomas. Youden index test was used to detect cut-off point, specificity, sensitivity, positive, and negative likelihood ratio.

Table 4 Area under the curve

\begin{tabular}{lc}
\hline Test result variable(s) & Area \\
\hline MRI_TI & .111 \\
MRI_T2 & .056 \\
MRI_DWI & .111 \\
MRI_DUAL & .056 \\
\hline
\end{tabular}

\section{Results}

This cross-sectional study included a total of 20 patients with adrenal lesions. Their ages ranged from 19 to 73 years with a mean value of $51.1 \pm 15.27$. All these patients were found to have either unilateral (18 patients) or bilateral (2 patients) adrenal lesions. Patients known to have a prior malignancy constitute $30 \%$ (6 patients) and patients with no history of known malignancy constitute $70 \%$ (14 patients) performing the exam due to other reasons like preoperative assessment of abdominal hernia repair, etc.

All the 22 lesions were subjected to size measurement along maximum axial dimensions. Positive group $(<4$ $\mathrm{cm}$ ) was formed of 13 lesions (59.09\%) and negative group (> $4 \mathrm{~cm}$ ) of 9 lesions (40.91\%). Benign adrenal lesions ranged in size from 1 to $10 \mathrm{~cm}$ with a mean of 3.05 while malignant lesions ranged from 2 to 6.5 with a mean of 4.64 .

Fig. 1 and Table 2 show the CT and MRI diagnosis of adrenal lesions.

Thirteen lipid-rich adenomas (59.1\%), 7 malignant looking lesions confirmed by a further tissue diagnosis (31.8\%) and 2 other lesions (9\%) including adrenal cyst and a myelolipoma. Six months follow-up MDCT and MRI studies were performed for all cases.

As shown in the ROC curve (Fig. 2) and area under the curve (Table 3), the CT_RPW are the best indicators 
Table 5 CT_DIAGNOSIS * MRI_DIAGNOSIS Cross-tabulation

\begin{tabular}{|c|c|c|c|c|c|c|c|}
\hline & & & \multicolumn{3}{|c|}{ MRI Diagnosis } & \multirow[t]{2}{*}{ Total } & \multirow[t]{2}{*}{$P$ value } \\
\hline & & & Adenoma & Malignant looking lesion & others & & \\
\hline \multirow[t]{4}{*}{ CT Diagnosis } & Adenoma & $\begin{array}{l}\text { Count } \\
\text { \%within }\end{array}$ & $\begin{array}{l}13 \\
100 \%\end{array}$ & $\begin{array}{l}0 \\
0.0 \%\end{array}$ & $\begin{array}{l}0 \\
0.0 \%\end{array}$ & $\begin{array}{l}13 \\
100 \%\end{array}$ & \multirow[t]{4}{*}{ Cannot be calculated } \\
\hline & Malignant looking lesion & $\begin{array}{l}\text { Count } \\
\text { \%within }\end{array}$ & $\begin{array}{l}0 \\
0.0 \%\end{array}$ & $\begin{array}{l}7 \\
100 \%\end{array}$ & $\begin{array}{l}0 \\
0.0 \%\end{array}$ & $\begin{array}{l}7 \\
100 \%\end{array}$ & \\
\hline & Others & $\begin{array}{l}\text { Count } \\
\text { \%within }\end{array}$ & $\begin{array}{l}0 \\
0.0 \%\end{array}$ & $\begin{array}{l}0 \\
0.0 \%\end{array}$ & $\begin{array}{l}2 \\
100 \%\end{array}$ & $\begin{array}{l}2 \\
100 \%\end{array}$ & \\
\hline & Total & & $\begin{array}{l}13 \\
100 \%\end{array}$ & $\begin{array}{l}7 \\
100 \%\end{array}$ & $\begin{array}{l}2 \\
100 \%\end{array}$ & $\begin{array}{l}22 \\
100 \%\end{array}$ & \\
\hline
\end{tabular}

used for CT detection of adrenal lipid-rich adenomas with sensitivity and specificity reaching $100 \%$.

As seen in Fig. 3, the ROC curve, and Table 4, area under the curve, we conclude that the MRI, CSI, and T2 signal are the best indicators used for MRI detecting intracytoplasmic fat content and confirming high lipid content in lipid-rich adenomas with a sensitivity of $100 \%$ and specificity of $88.89 \%$.

Table 5 shows that a similar diagnostic outcome is obtained from CECT and MRI with CSI of adrenal lesions with a net result of 13 typical lipid-rich adenomas (Fig. 4), 7 malignant lesions (Figs. 5 and 6), 1 simple adrenal cyst (Fig. 7), and 1 suprarenal myelolipoma (Fig. 8).

\section{Discussion}

Unexpectedly discovered adrenal masses are found in about $5 \%$ of all abdominal CT examinations done in patients not known to have malignancy or endocrine abnormality. The incidence of AIs increases to $9-13 \%$ in patients imaged for a known malignancy [8].

The most common AI is benign adrenal adenoma [9]. The aim of this work is to study the role of MRI, specifically chemical shift imaging (CSI), against various MDCT scans (non-enhanced, enhanced, and delayed) in the characterization of incidentally discovered adrenal masses to offer a way for the patients to avoid unnecessary time and money-wasting imaging modalities used to reach a diagnosis of their incidentally discovered adrenal lesions.

In previous studies, a considerable overlap in size between the suprarenal adenomas and the nonadenomatous lesions, especially metastases was demonstrated. This was also noted in our study. We found that the size of $<4 \mathrm{~cm}$ has $100.00 \%$ sensitivity and $66.6 \%$ specificity for being a benign suprarenal adenoma. This is keeping with Angelelli et al. 2013 stating that the size
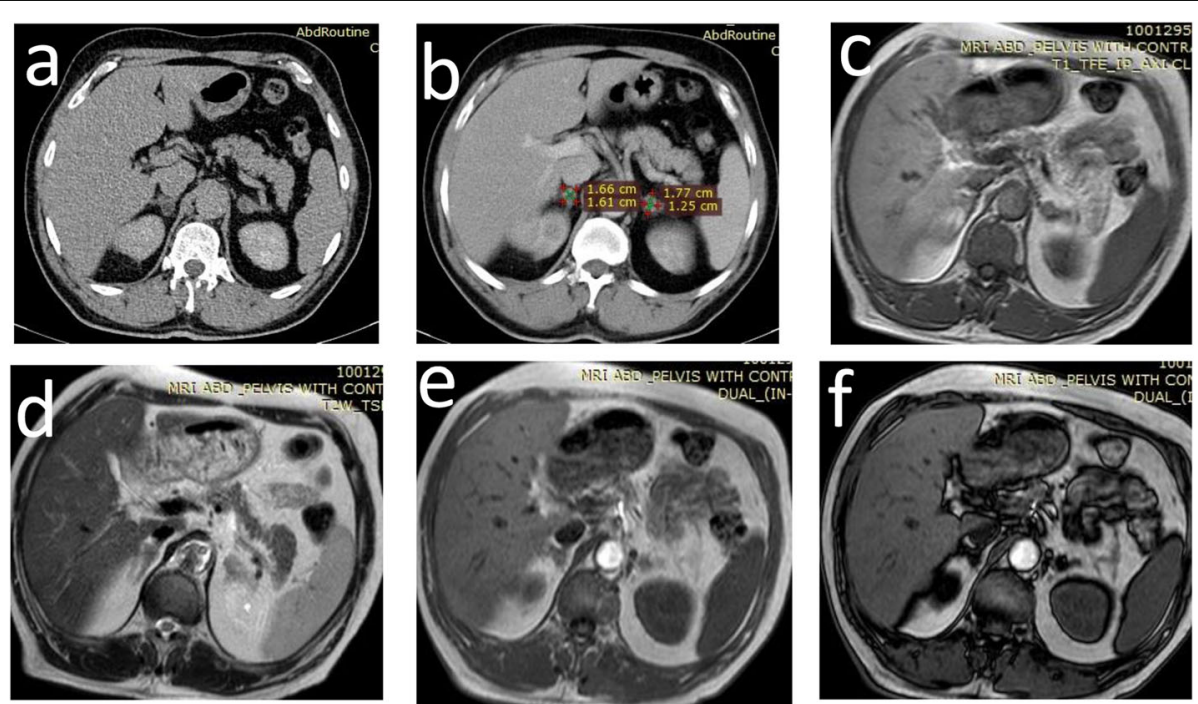

Fig. 4 A 60-year-old male patient complaining of progressive hematuria was diagnosed by cystoscopy and histopathology to have bladder carcinoma. a Precontrast and $\mathbf{b}$ Post-contrast MDCT abdomen axial images showing bilateral hypodense suprarenal nodules measuring $1.6 \mathrm{~cm}$ on the right side and $1.7 \mathrm{~cm}$ on the left one along their maximum diameter. RPW $=63 \%$ on both sides. MRI suprarenal protocol (c) axial T1 (d) axial T2 showing bilateral suprarenal nodules of intermediate signal in both sequences. $\mathbf{e}$, $\mathbf{f}$ in phase and out of phase images showing differential and remarkable loss of signal in the opposed phase images denoting intracytoplasmic fat, along with their small size favoring the diagnosis of lipidrich adenomas. Diagnosis was further proven by PET CT showing no suprarenal metastasis 

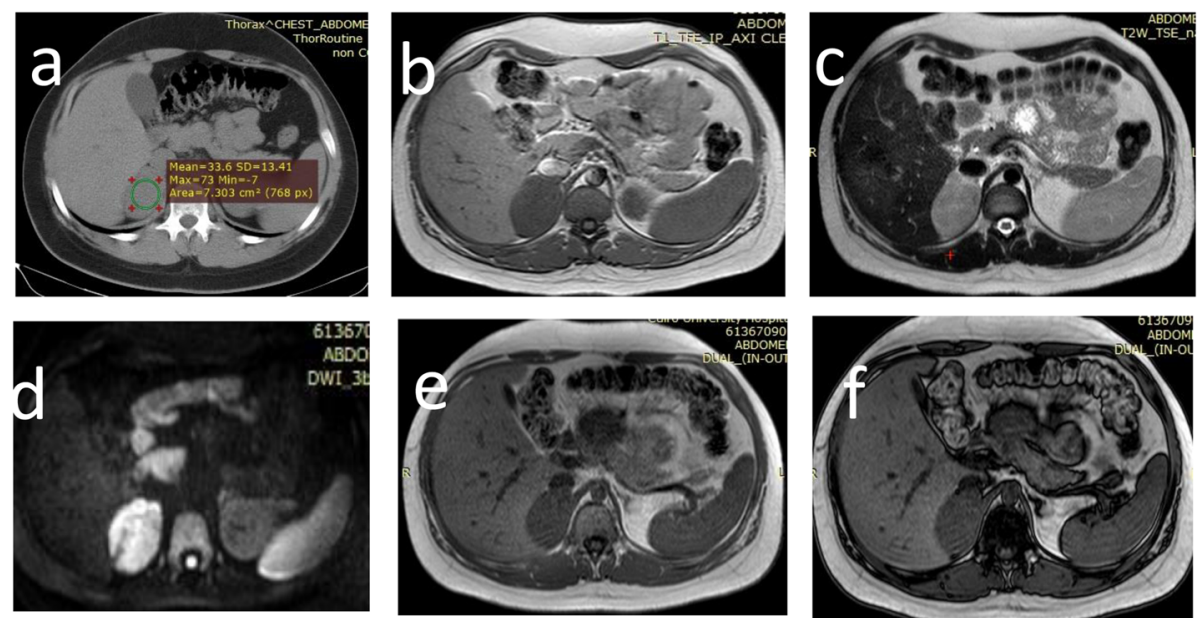

Fig. 5 A 19-year-old male patient with a brain SOL that was surgically removed and sent to pathology and diagnosed as lymphoma a MDCT axial cuts showing right suprarenal soft tissue mass lesion measuring $6.2 \times 4.8 \mathrm{~cm}$ with a mean attenuation of $33 \mathrm{HU}$ in unenhanced images. APW $=22 \%$ and RPW $=4 \%$ denoting poor lipid content. MRI abdomen suprarenal protocol showing right suprarenal soft tissue mass lesion of iso T1 signal (b), intermediate T2 signal (c), it shows diffusion restriction in $\mathbf{d}$ and no drop of signal in opposed phase images (f) compared to in phase 1 (e). Diagnosis was right suprarenal metastatic lesion
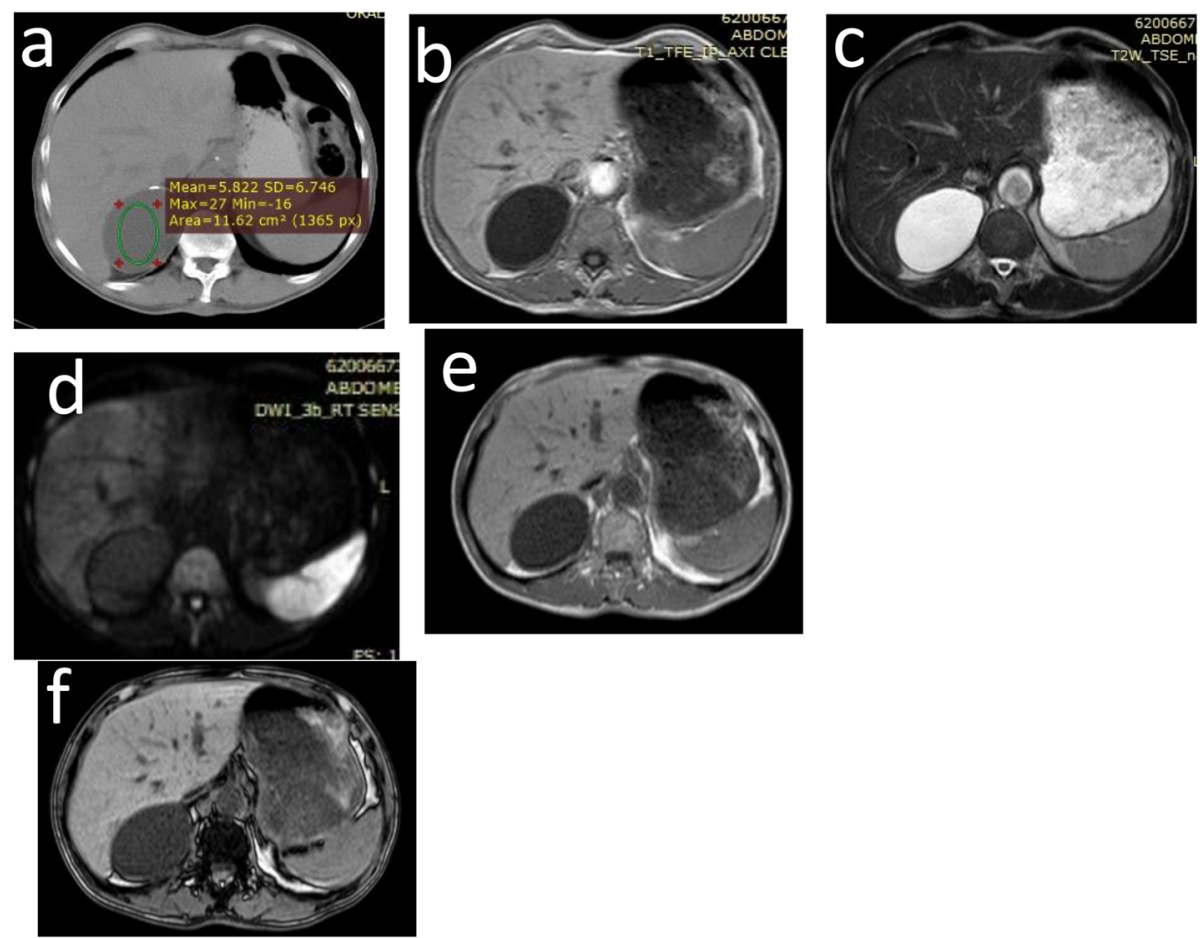

Fig. 6 A 50-year-old female patient came to the ER then referred to Urology Department complaining of left loin pain of gradual onset and progressive course over the past few months. a Axial non-enhanced CT of the abdomen showing a rather large soft tissue mass lesion measuring $6.6 \times 5.2 \mathrm{~cm}$ along maximum axial dimensions implicating the right suprarenal gland region with mean attenuation of $19 \mathrm{HU}$ in unenhanced images. MRI of abdomen adrenal protocol showing a large-sized right suprarenal soft tissue mass lesion eliciting intermediate signal in both T1 (b) and T2Wls (c), bright signal in diffusion (d) denoting restricted diffusion and showing no signal drop from the in phase to the out phase images (e, f). Diagnosis was right suprarenal malignant neoplastic lesion and was confirmed pathologically to be an adreno-cortical adenocarcinoma 

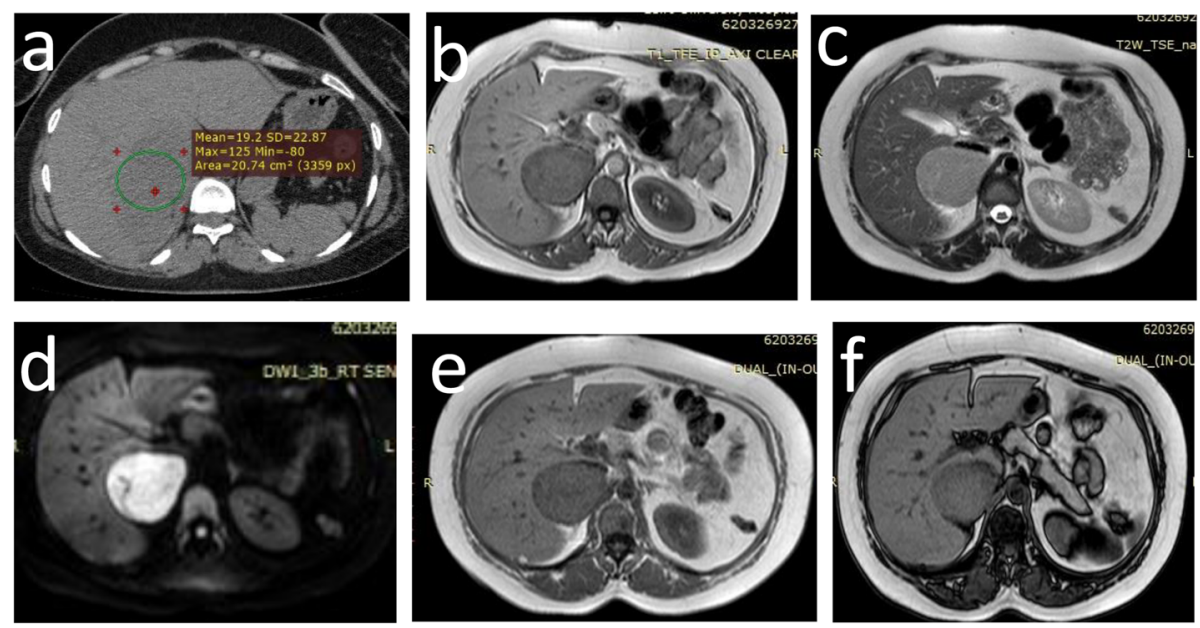

Fig. 7 A 63-year-old male patient complaining of occasional right upper abdominal pain of long duration. a CECT of the abdomen showing right thin walled cystic lesion of the right suprarenal gland measuring $7 \mathrm{~cm} \times 6 \mathrm{~cm}$ in maximum axial dimensions with no solid component or significant mural enhancement with internal homogenous fluid content $5 \mathrm{HU}$. MRI abdomen adrenal protocol showing right suprarenal cystic lesion eliciting low TI signal (b) and bright T2 signal (c). No diffusion restriction (d) eliminating the possibility of an abscess. No signal drop in the opposed phase image $(f)$ in relation to in phase image (e). Diagnosis: right simple suprarenal cyst, no further evaluation was required

criterion yielded $98 \%$ sensitivity, but a specificity of $53 \%$, which was too low to be accepted as a discriminator [6]. We concluded that the differentiation between benign and malignant suprarenal lesions cannot rely on the lesions size alone. The size could be a helpful factor to reach the precise diagnosis but still we need more reliable diagnostic criteria.

In our study, we demonstrated that unenhanced CT (UCT) attenuation value of adrenal lesions is a useful imaging modality to characterize lipid-rich adenomas that measure $10 \mathrm{HU}$ or less. A threshold of $10 \mathrm{HU}$ on UCT can be used. The sensitivity and specificity for adenoma characterization were $72.7 \%$ and $77.78 \%$, respectively.
This partially agrees with Park et al. 2016 and Kamiyama et al. 2009 who came out with that an attenuation value of a suprarenal mass of less than $10 \mathrm{HU}$ has a sensitivity and specificity for adenoma of $71 \%$ and $98 \%$, respectively $[10,11]$.

In this study, the unenhanced value is considered significant to distinguish between adenomas and nonadenomas. This is keeping with Angelelli et al. 2013 stating that no case of non-adenoma lesion with the exception of myelolipomas and cysts has a basal density of less than $10 \mathrm{HU}$ [6].

Previous investigators have demonstrated that the washout attenuation is much larger in adenomas than in non-adenomas $[12,13]$. In our study, washout values
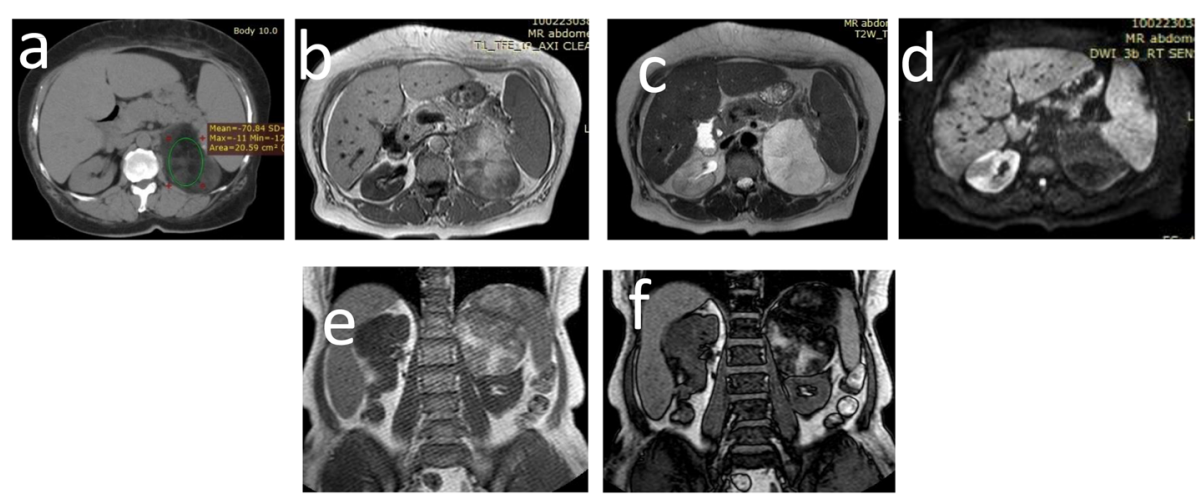

Fig. 8 A 50-year-old female patient known SLE with high creatinine level due to associated nephropathy came for a CTUT. a NECT of abdomen axial section showing left suprarenal soft tissue mass lesion measuring $10 \mathrm{~cm}$ in maximum axial dimension with heterogeneous attenuation with areas of pure fat content giving attenuation of - $70 \mathrm{HU}$. MRI abdomen adrenal protocol showing left suprarenal large soft tissue lesion with areas of high T1 (b) and less bright T2 signal (c) with facilitated diffusion (d). These areas show marked drop of signal. Diagnosis: left

suprarenal myelolipoma 
were also significantly larger in lipid-rich adenomas. We concluded that relative percentage washout (RPW) is a good indicator of the lipid-rich content and benignity of adenomas with area under the curve (AUC) 1.000, sensitivity and specificity of $100 \%$ (Table 2 ) in comparison to absolute percentage washout (APW) with (AUC) 0.883, sensitivity and specificity of $90 \%$ and $85 \%$, respectively.

This agrees with Park et al. 2016 who stated that RPW is more accurate than APW for diagnosing an adrenal adenoma. If an adenoma measures less than $0 \mathrm{HU}$ in unenhanced CT images, RPW becomes higher than APW. Therefore, it is not uncommon for APW to be below $60 \%$ in lipid-rich adenoma [10].

The best MR imaging technique for to characterize suprarenal adenoma is found to be chemical shift imaging (CSI). Its sensitivity and specificity in diagnosis of indeterminate adrenal lesions are reported as $67 \%$ and $89-100 \%$, respectively [7]. Subgroup analysis reveals that for adrenal lesions measuring 10-30 HU in unenhanced CT studies, the sensitivity of CSI is as high as $89 \%$, which is comparable to the data retrieved from washout CT. However, its sensitivity declines markedly to be as low as $13 \%$ for suprarenal lesions measuring more than $30 \mathrm{HU}$ in unenhanced CT images. This concludes that MR imaging is not a reliable tool for these lesions, although the specificity can be as high as $100 \%$ [14]. On that account, adrenal lesions measuring 10-30 HU can be further evaluated with either washout $\mathrm{CT}$ or chemical shift MR imaging according to personal and institutional circumstances; lesions with $\mathrm{CT}$ attenuation measuring higher than $30 \mathrm{HU}$ are best imaged with washout CT [7].

In our study, CSI, also referred to by dual phase imaging, has proven to be the best indicator for evaluating adenomas from non-adenomas, showing loss of signal in all benign lesions except for adrenal cysts with a total of $14 / 15$ benign lesions. Drop of signal in the out phase images showing the highest (AUC) 0.944 a standard error of 0.0556 with sensitivity and specificity of $100 \%$ and $88.89 \%$, respectively.

This agrees with El-Kalioubie et al. 2016 who stated that diagnosis of malignant lesions was accurately reached by observing the lack of signal drop on CS MRI in all malignant lesions, by showing the absence of signal drop on out-of-phase (OP) images and showing signal loss in all lipid-rich lesions [15]. There was no signal loss in any of the malignant masses. Some adrenocortical carcinomas have been reported to contain intracellular lipid [16]; however, no cases of lipid-rich adrenocortical carcinomas were included in our study population.

\section{CT versus CS MRI}

No statistically significant difference was seen in our study regarding the preference of using CT over CSI
(Table 4) unless in certain situations when the use of one diagnostic modality may be superior.

This is keeping with literature stating that the sensitivity and specificity of CSI for the differentiation of incidental adrenal lesion are similar to those of unenhanced CT densitometry, at $81-100 \%$ and $94-100 \%$, respectively $[14,17,18]$.

Studies have shown that for lipid-rich adenomas, there is effectively no difference between CECT and MRI, as in our study, but CSI might be superior when evaluating lipid-poor adenomas [14]. However, Park et al. 2016 showed that CSI might be useful only when the unenhanced CT attenuation is less than $30 \mathrm{HU}$ and that delayed enhanced CT can characterize additional hyperattenuating adrenal masses that cannot be identified with chemical shift MRI [10].

\section{Limitations}

Some limitations that were encountered in our study included our study design which depended on accidentally discovered adrenal masses in patients undergoing abdominal studies for irrelevant causes which limited our sample size. Also, other limitations included the wide varieties of pathologies that cause incidentally discovered adrenal masses. This variation affected the sample size in each pathological category. Further study on a larger number of patients may be of value in retrieving better statistical results.

\section{Conclusion}

There is no statistically significant difference in the diagnostic capability of the CSI versus the CT washout technique. Both modalities could be conducted according to specific patient preferences and/or limitations with comparable highly accurate outcomes.

\section{Abbreviations}

Al: Adrenal incidentaloma; ACC: Adreno-cortical carcinoma; APW: Absolute percentage washout; CECT: Contrast enhanced tomography; CSI: Chemical shift imaging; DWl: Diffusion-weighted imaging; HU: Hounsfield unit; KV: Kilo voltage; mA: Milli-amber; MDCT: Multi-detector computed tomography; MRl: Magnetic resonance imaging; ms: Milliseconds; PET: Positron emission tomography; ROC: Receiver operating characteristic; ROI: Region of interest; RPW: Relative percentage washout; T1Wls: T1-weighted images; T2Wls: T2weighted images; TE: Echo time; TR: Repetition time; UCT: Unenhanced computed tomography

\section{Acknowledgements}

The authors would like to thank all those who contributed to this study.

\section{Authors' contributions}

S.S. contributed to the study's conception and design, collected patients' data, processed CT findings at the CT workstation, and shared in writing and revising the manuscript. M.N. shared in the study's conception and design; acquired, analyzed, and interpreted data; and contributed to the drafting of the manuscript. M.N. did the evaluation and made the post-processing for both modalities. S.H. and S.E. shared in the study's conception and design, conducted clinical examinations, acquired, analyzed and interpreted data, and contributed to the drafting of the manuscript. All authors mentioned read and approved the final manuscript. 


\section{Funding}

This study was not funded by any resource.

\section{Availability of data and materials}

The sets of data used and/or analyzed during the study are available upon sensible request.

\section{Ethics approval and consent to participate}

No individual data was included in the study. This study was approved by the Research Ethics Committee of the Faculty of Medicine at Cairo University Kasr El-Aini in Egypt in May 2019; reference number MS-129-2019. All patients participating in this study were informed of what it entails and gave informed consent to take part in this research. If the patient was unconscious at the time of the study, written informed consent for their participation was provided by their legal guardian.

\section{Consent for publication}

All patients included in this study gave written informed consent to publish the data obtained within this study.

If the patient was unconscious when consent for publication was requested, written informed consent for the publication was provided by their legal guardian.

\section{Competing interests}

The authors of this study declare that they have no competing interests.

\section{Author details}

'Department of Radiodiagnosis, Faculty of Medicine, Cairo University, Cairo, Egypt. ${ }^{2}$ Department of Internal Medicine, Faculty of Medicine, Cairo University, Cairo, Egypt.

Received: 9 November 2020 Accepted: 7 February 2021

Published online: 19 February 2021

\section{References}

1. Grumbach MM, Biller BM, Braunstein GD et al (2003) Management of the clinically inapparent adrenal mass (incidentaloma). Ann Intern Med 138(5): 424-429

2. Chatzellis E., and Kaltsas G. (2019) Adrenal incidentalomas. In: Feingold KR, Anawalt B, Boyce A, et al., editors. Endotext [Internet]. South Dartmouth (MA): MDText.com, Inc.; Available from: https://www.ncbi.nlm.nih.gov/ books/NBK279021/

3. Schieda N, Siegelman ES (2017) Update on CT and MRI of adrenal nodules. AJR Am J Roentgenol 208(6):1206-1217. https://doi.org/10.2214/ajr.16.17758

4. Zeiger MA, Thompson GB, Hamrahian AH et al (2009) American Association of Clinical Endocrinologists and American Association of Endocrine Surgeons medical guidelines for the management of adrenal incidentalomas. Endocr Pract 15:1. https://doi.org/10.4158/ep.15.5.450

5. Lau J, Balk E, Rothberg M, loannidis JP et al (2002) Management of clinically inapparent adrenal mass. Evid Rep Technol Assess (Summ) (56):1

6. Angelelli G, Mancini ME, Moschetta M et al (2013) MDCT in the differentiation of adrenal masses: comparison between different scan delays for the evaluation of intralesional washout. Scientific World Journal:1-7. https://doi.org/10.1155/2013/957680

7. Adam SZ, Nikolaidis P, Horowitz JM et al (2016) Chemical shift MR imaging of the adrenal gland: principles, pitfalls, and applications. Radiographics 36(2):414-432

8. Bovio S, Cataldi A, Reimondo G et al (2006) Prevalence of adrenal incidentaloma in a contemporary computerized tomography series. J Endocrinol Invest 29:298-302. https://doi.org/10.1007/BF03344099

9. Garrett RW, Nepute JC, Hayek ME et al (2016) Adrenal incidentalomas: clinical controversies and modified recommendations. American Journal of Roentgenology (AJR) 206(6):1170-1178. https://doi.org/10.2214/ajr.15.15475

10. Park JJ, Park BK, Kim CK (2016) Adrenal imaging for adenoma characterization: imaging features, diagnostic accuracies and differential diagnoses. Br J Radiol 89(1062):20151018. https://doi.org/10.1259/bjr.20151 018

11. Kamiyama T, Fukukura Y, Yoneyama T et al (2009) Distinguishing adrena adenomas from nonadenomas: combined use of diagnostic parameters of unenhanced and short 5-minute dynamic enhanced CT protocol. Radiology 250(2):474-481. https://doi.org/10.1148/radiol.2502080302
12. Szolar DH, Kammerhuber FH (1998) Adrenal adenomas and nonadenomas: assessment of washout at delayed contrast-enhanced CT. Radiology 207(2): 369-375. https://doi.org/10.1148/radiology.207.2.9577483

13. Caoili EM, Korobkin M, Francis IR et al (2000) Delayed enhanced CT of lipidpoor adrenal adenomas. American Journal of Roentgenology (AJR) 175(5): 1411-1415. https://doi.org/10.2214/ajr.175.5.1751411

14. Haider MA, Ghai S, Jhaveri K et al (2004) Chemical shift MR imaging of hyperattenuating $(>10 \mathrm{HU})$ adrenal masses: does it still have a role? Radiology 231(3):711-716. https://doi.org/10.1148/radiol.2313030676

15. El-Kalioubie M, Emad-Eldin S, Abdelaziz O (2016) Diffusion-weighted MRI in adrenal lesions: A warranted adjunct? The Egyptian Journal of Radiology and Nuclear Medicine 47(2):599-606. https://doi.org/10.1016/j.ejrnm.2016.01. 003

16. Yamada T, Saito H, Moriya T et al (2003) Adrenal carcinoma with a signal loss on chemical shift magnetic resonance imaging. J Comput Assist Tomogr 27(4):606-608

17. Koo HJ, Choi HJ, Kim HJ et al (2014) The value of 15-minute delayed contrast-enhanced CT to differentiate hyperattenuating adrenal masses compared with chemical shift MR imaging. Eur Radiol 24(6):1410-1420. https://doi.org/10.1007/s00330-013-3084-7

18. Israel GM, Korobkin M, Wang C et al (2004) Comparison of unenhanced CT and chemical shift MRI in evaluating lipid-rich adrenal adenomas. American Journal of Roentgenology (AJR) 183(1):215-219. https://doi.org/10.2214/ajr.1 83.1.1830215

\section{Publisher's Note}

Springer Nature remains neutral with regard to jurisdictional claims in published maps and institutional affiliations.

\section{Submit your manuscript to a SpringerOpen ${ }^{\circ}$ journal and benefit from:}

- Convenient online submission

- Rigorous peer review

- Open access: articles freely available online

High visibility within the field

- Retaining the copyright to your article

Submit your next manuscript at $\boldsymbol{\nabla}$ springeropen.com 